\title{
A Mathematical Modeling for Delivery Time in Dynamic PERT Networks
}

\author{
Mahtab Afsari ${ }^{1} \&$ Hesam Javadi Vasigh ${ }^{2}$ \\ ${ }^{1} \mathrm{PhD}$ student, Institute of Product Development, Leibniz University Hannover, Germany \\ ${ }^{2}$ Msc in Industrial Management \\ Correspondence: Mahtab Afsari, Institute of Product Development, Leibniz University Hannover, Germany. \\ E-mail: afsari@ipeg.uni-hannover.de
}

Received: January 15, 2016

doi:10.5539/mas.v10n3p214
Accepted: February 1, 2016

Online Published: February 29, 2016

URL: http://dx.doi.org/10.5539/mas.v10n3p214

\begin{abstract}
In project management, the main purpose of proposed methods is project scheduling which helps to management and planning. We can calculate a real average of project closure in dynamic PERT networks by identifying a lower limit. According to randomness, each distribution parameter varies itself and also it is proposed that activity durations are exponential distributions which have casual, unstable and independent variables. We used hypothetic kinetic programming, that project closure time would have a lower limit. Finally, delivery time would obtain optimal due date by minimizing the expected aggregate cost per project and also a linear function of its due-date.
\end{abstract}

Keywords: dynamic PERT networks, delivery time, mathematical modeling

\section{Introduction}

Chanas and Kamburowski introduced a Fuzzy logic approach for project management in 1970s. They used evaluation and review method which was based on fuzzy (Lachmayer \& Afsari, 2012). In recent years, a project-based approach, has been developed with considering the organization's activities (Akbari et al, 2011). Ying et al. 2009, showed that in these situations, there are several projects simultaneously for employing resources and their share in the project.

As it presented in (Lachmayer \& Afsari, 2012), uncertainties and probabilities have become essential concepts of human life. With regard to this point that Probability theory is able to model uncertainties with random identification only, in other words, it cannot model indeterminacy of incomplete information. Project activities are along with the uncertainties that may cause many irregularity. On the other hand, some organizations have multiple projects, in addition to uncertainty in the duration of projects activities which may entre dynamically, consequently makes the planning process complicated (Abdelkader, 2004). Furthermore, dynamic PERT networks including additional stuff and activities during the projects are considered as dynamic environment which results in significant uncertainties (Yaghoubi et al, 2011). Therefore, project scheduling should be considered as one of the integral parts of project management, which can be described as all of the following:

- Management - in organization activity is the act of getting people together to achieve desired objectives. Management comprises planning, organizing, staffing, leading or directing, and controlling an organization or effort for the purpose of accomplishing a goal.

- Management process - is a process of planning and controlling the performance or execution of any type of activity.

- $\quad$ Process - ongoing collection of activities, with inputs, outputs and the energy required to transform inputs to outputs.

- $\quad$ Project - A temporary endeavor undertaken to create a unique product, service, or result

So, a project manager should be able to handle various subjects such as cost management, scheduling, quality and resource management. Proper project staffing is critical, yet improperly allocating resources tops the list of most common project management mistakes. Project managers need full visibility into the skills and workloads of all of their resources, including consultants, contractors and outsourcers, who often get left out of skills 
assessments even though they're doing a "huge" proportion of work. This issue was studied using the process approach, so that organization was considered as a probable processing network with multiple workstations. Abdelkader [1] has used moment methods for estimating project completion time based on Weibull project distribution time. Elmaghraby [7] stated the changes of the mean and variance related to the importance of standard deviation considered project completed time.

Cohen et al [6] developed a new method for analyzing the completed project time with considering the time which expend for each activity.

Most recently assigned to the deadline for submission of the dynamic PERT networks based on the assumption, be unlimited servers in each workstation and infinite capacity of Azaron et al system. [3] It should be noted that in this paper we study the generalized Azaron's study for project allocation in dynamic PERT networks with multiple servers and unlimited capacity will be case by a Markov chain with a number of states bound and continuous-time (Azaron et al, 2008). In this paper, a real average of project closure in dynamic PERT networks are calculated by identifying a lower limit. For this purpose, a combined novel semi-Markovian processes and graph theory is developed.

\section{Project evaluation and review technique networks of Dynamic Markov}

By using dynamic Markov method, a project evaluation and review technique network is considered as a $\mathrm{G}$ function $(\mathrm{G}=(\mathrm{W}, \mathrm{B}))$, where, $\mathrm{W}$ and $\mathrm{B}$ represent $G$ nodes on activities. Then $\mathrm{N}$ is the number of effective social randomness and, $\left(\mathrm{s}^{1}, \mathrm{~s}^{2}, \ldots, \mathrm{s}^{\mathrm{N}}\right)$ represent the state vectors of system in each node $l \in V$, based on activities originating from node $l$.it is assumed that activity duration $(1, \mathrm{j}) \in A$ is stochastic parameter with $\chi_{\mathrm{lj}}\left(\mathrm{s}^{1}, \mathrm{~s}^{2}, \ldots\right.$, $\mathrm{s}^{\mathrm{N}}$ ), which is the function of the state vector in node 1. According Markov procedure, Social randomness developed over the time horizon. We considered following assumption:

1. $\mathrm{N}_{\mathrm{i}}$ stands for the Number of states of $i$ th social randomness (variables order $\left(s_{1}^{i}, s_{2}^{i}, \ldots, s_{N}^{i}\right)$, and $P_{m k}^{i}$ represents the expectance of social randomness from state $s_{m}^{i}$ to state $s_{k}^{i}$.

2. Let $t_{m k}^{i}$ represent the transition time from state $s_{m}^{i}$ to state $s_{k}^{i}$.

Considering $t_{m}^{i}$ as the staying time of $i$ th social randomness in state $s_{m}^{i}$, the density function or $w_{m}^{i}$ would be as follow [15]:

$$
w_{m i}^{i}(\mathrm{t})=\sum_{k=1}^{N} P_{m k}^{i} f_{m k}^{i}(\mathrm{t})
$$

3. A Markov process is a stochastic process which satisfies the condition that the future

depends only on the present and not on the past. It can be shown that a finite state irreducible Markov chain is ergodic if it has an aperiodic state. A model has the ergodic property if there's a finite number $\mathrm{N}$ such that any state can be reached from any other state in exactly $\mathrm{N}$ steps.

4. Let $\emptyset_{m k}^{i}$ demonstrate the conditional status, where $i$ th social randomness input in state $s_{k}^{i}$ at time t, given that it was in state $s_{m}^{i}$ at time zero (Fatemi et al, 2003).

How can a process that started by entering state $s_{m}^{i}$ at time zero be in state $s_{k}^{i}$ at time $t$ ? One way is that, this is for $s_{m}^{i}$ to $s_{k}^{i}$ in order to be as the previous state, therefore the process doesn't loose state $s_{m}^{i}$ in the period $(0, \mathrm{t})$. Any method to get from state $s_{m}^{i}$ to state $s_{k}^{i}$ in the interval $(0, \mathrm{t})$ requires making at least one transition during that interval For example, the process could make its first transition from state $s_{m}^{i}$ some state $s_{k}^{i}$ at a time $\tau, 0<\tau<\mathrm{t}$, and then, by some succession of transitions have made its way to state $s_{k}^{i}$ at time t. These considerations lead us to Equation (2) for computing $\emptyset_{m k}^{i}$

$$
\emptyset_{m k}^{i}(\mathrm{t})=\delta_{m k} \int_{t}^{\infty} w_{m}^{i}(\tau) \mathrm{d} \tau+\sum_{I=1}^{N} f_{m I}^{i}(\tau) \emptyset_{I k}^{i}(\mathrm{t}-\tau) \mathrm{d} \tau, \quad \delta_{m k}=\left\{\begin{array}{cc}
1 & \text { if } m=k \\
0 & \text { otherwise }
\end{array}\right.
$$

Certainly, we cannot directly calculate $\emptyset_{m k}^{i}$ from Equation. (2), but since the second integral of Equation. (2) Is a convolution of two functions, we can easily calculate $\emptyset_{m k}^{i}(\mathrm{t})$ by the Laplace transform. Let $f_{m l}^{i}(s)$ and $\emptyset_{m k}^{i}(\mathrm{~s})$ Is the Laplace transforms of $f_{m l}^{i}(t)$ and $\emptyset_{m k}^{i}(\mathrm{t}) . \emptyset_{m k}^{i}(\mathrm{~s})$ is given by Equation (3) (Azaron et al, 2008):

$$
\emptyset_{m k}^{i c}(\mathrm{~s})=\delta_{m k} \iint e^{-s t} w_{m}^{i}(\tau) \mathrm{d} \tau \mathrm{dt}+\sum_{I=1}^{N} P_{M I}^{i} f_{m I}^{i}(\mathrm{~s}) \emptyset_{I k}^{i}(\mathrm{~s})
$$

Markov process with finite state if (E,F) are PTC acceptable (Yaghoubi et al, 2011). 


$$
\begin{aligned}
& q\left\{(E, F),\left(E^{\prime}, F^{\prime}\right)\right\}=\left\{\begin{array}{cl}
\gamma_{a} & \text { if }: I \\
\gamma_{a} & \text { if }: \text { II } \\
-\sum_{a \in E} \gamma_{a} & \text { if }: \text { III } \\
0 & \text { otherwise }
\end{array}\right. \\
& I: a \in E, I(\beta(a)) \subset F \cup\{a\}, E^{\prime}=E-\{a\}, F^{\prime}=F \cup\{a\} \\
& \text { II }: a \in E, I(\beta(a)) \subset F \cup\{a\}, E^{\prime}=(E-\{a\}) \cup O(\beta(a)), F^{\prime}=F-I(\beta(a)) \\
& \text { III }: a \in E, I(\beta(a)) \subset F \cup\{a\}, E^{\prime}=(E-\{a\}) \cup O(\beta(a)), F^{\prime}=F-I(\beta(a))
\end{aligned}
$$

Let $\mathrm{P}(\mathrm{t})$ represent the conditional status of parameter $(1, \mathrm{j})$ (Kumanan et al, 2006),

$$
\mathrm{P}(\mathrm{t})=\mathrm{M} \cdot \mathrm{e}^{\Delta \mathrm{t}} \cdot \mathrm{M}^{-1} \cdot \mathrm{P}(0)
$$

That,

$$
e^{\Delta t}=\left[\begin{array}{ccc}
e^{\alpha 1 t} & \cdots & 0 \\
\vdots & \ddots & \vdots \\
0 & \cdots & e^{\propto N t}
\end{array}\right]
$$

Let A (l) be the set of forward adjacent nodes of node 1. According to the proposed algorithm, first calculate $\mathrm{F}(\mathrm{B})$ with use of Eq.5, Second, if $F(B)>(K 2 /(K 2+K 3))$ then $t^{*}=\bar{t}(d)$ whatever $F(\bar{t}(d))=(K 2 /(K 2+K 3))$ else [16]:

if $F(B)<((K 2-K 1) /(K 2+K 3))$ then $t^{*}=\bar{t}(d)$ whatever $F(\bar{t}(d))=((K 2-K 1) /(K 2+K 3))$ and $t^{*}=B$.

\section{Data Analysis}

The population studied in this research, Provision of study materials required for the production of concrete plants in Iran. Figure 1 shows of dynamic PERT network into a graph diagram.

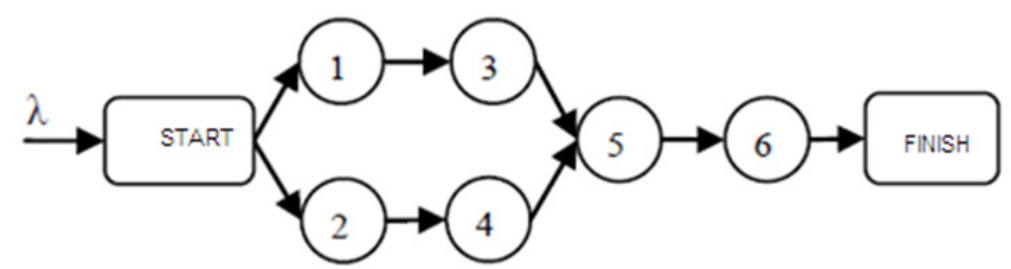

Figure 1. Dynamic PERT network

Table 1 presents their values, and lower bonds are considered as:

$$
\begin{aligned}
& f_{11}^{1}(\mathrm{t})=f_{12}^{1}(\mathrm{t})=f_{21}^{1}(\mathrm{t})=f_{22}^{1}(\mathrm{t})=e^{-t}, \mathrm{t}>0 \\
& f_{11}^{2}(\mathrm{t})=4 e^{-4 t}, \mathrm{t}>0 \\
& f_{12}^{2}(\mathrm{t})=2 e^{-2 t}, \mathrm{t}>0 \\
& f_{21}^{2}(\mathrm{t})=3 e^{-3 t}, \mathrm{t}>0 \\
& f_{22}^{2}(\mathrm{t})=e^{-t}, \mathrm{t}>0
\end{aligned}
$$

Table 1. Parameters of the activity durations

\begin{tabular}{|l|c|c|c|c|c|c|c|}
\hline & $\lambda_{11}$ & $\lambda_{14}$ & $\lambda_{24}$ & $\lambda_{25}$ & $\lambda_{35}$ & $\lambda_{36}$ & $\lambda_{46}$ \\
\hline$\left(s_{1}^{1}, s_{1}^{2}\right)$ & 1 & 2 & 2 & 4 & 1 & 2 & 5 \\
\hline$\left(s_{1}^{1}, s_{2}^{2}\right)$ & 1 & 2 & 3 & 5 & 3 & 3 & 5 \\
\hline$\left(s_{2}^{1}, s_{1}^{2}\right)$ & 5 & 5 & 8 & 8 & 4 & 6 & 9 \\
\hline$\left(s_{2}^{1}, s_{2}^{2}\right)$ & 5 & 4 & 8 & 9 & 6 & 7 & 9 \\
\hline
\end{tabular}


Table 2. $V_{1}\left(s_{m 1}^{1}, s_{m 2}^{2}\right)$ for $\mathrm{i}=1,2, m_{i}=1,2$ and $l=1,2,3,4$

\begin{tabular}{|c|c|c|c|c|}
\hline & $V_{1}$ & $V_{2}$ & $V_{3}$ & $V_{4}$ \\
\hline$\left(s_{1}^{1}, s_{1}^{2}\right)$ & 1.86 & 1.281 & 1.179 & 0.28 \\
\hline$\left(s_{1}^{1}, s_{2}^{2}\right)$ & 1.3 & 0.698 & 0.427 & 0.158 \\
\hline$\left(s_{2}^{1}, s_{1}^{2}\right)$ & 0.912 & 0.618 & 0.459 & 0.132 \\
\hline$\left(s_{2}^{1}, s_{2}^{2}\right)$ & 0.612 & 0.379 & 0.254 & 0.09 \\
\hline
\end{tabular}

In this study, computational results for proposed method are presented in table 3. Based on these results, It was concluded from results that the maximum percentage difference is about $13 \%$. So, it indicates that, in this dynamic PERT networks case study, approximation of proposed method is near to lower bound for the true mean project completion time.

Table 3. Computational results

\begin{tabular}{|c|c|c|c|}
\hline Number of nodes & Number of activities & Maximum in-degree & Percentage difference \\
\hline 3 & 4 & 2 & 5.71 \\
\hline 6 & 8 & 2 & 9.98 \\
\hline 8 & 11 & 2 & 8.59 \\
\hline 9 & 13 & 3 & 10.53 \\
\hline 10 & 17 & 4 & 12.27 \\
\hline
\end{tabular}

$\emptyset_{11}^{1}(\mathrm{t})=0.27+0.68 \mathrm{e}^{-1.2 \mathrm{t}}, \emptyset_{12}^{1}=1-\emptyset_{11}^{1}(\mathrm{t})$

$\emptyset_{21}^{1}(\mathrm{t})=0.27+0.32 \mathrm{e}^{-1.2 \mathrm{t}}, \emptyset_{22}^{1}=1-\emptyset_{21}^{1}(\mathrm{t})$

$\emptyset_{11}^{2}(\mathrm{t})=0.39+0.76 \mathrm{e}^{-0.91 \mathrm{t}}-0.07 \mathrm{e}^{-2.78 \mathrm{t}}, \emptyset_{11}^{2}=1-\emptyset_{11}^{2}(\mathrm{t})$

$\emptyset_{21}^{2}(\mathrm{t})=0.39+0.08 \mathrm{e}^{-0.91 \mathrm{t}}-0.27 \mathrm{e}^{-2.78 \mathrm{t}}, \emptyset_{22}^{2}=1-\emptyset_{21}^{2}(\mathrm{t})$

Figure 2 shown that Rate of Markov Chain with all PTC acceptable for this problem:

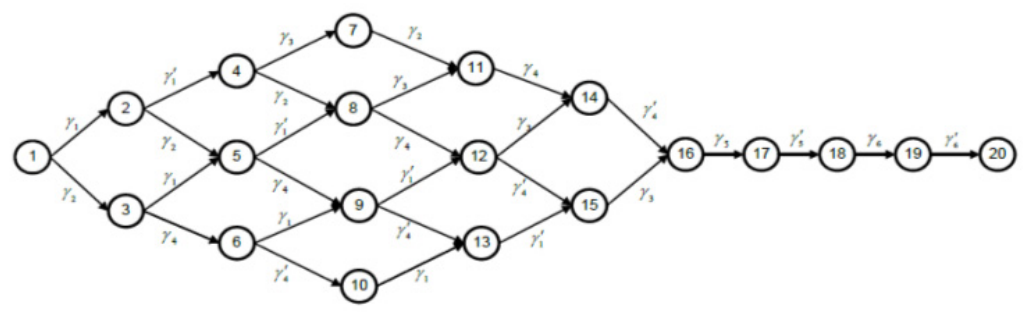

Figure 2. Rate of Markov Chain with continuous-time

Accordingly,

$$
\begin{gathered}
\gamma_{a}=\frac{\left(m_{a} \mu_{a}-\lambda\right) \cdot m_{a} \mu_{a}}{\chi} \\
\gamma_{a}^{\prime}=\frac{m_{a} \mu_{a}}{m_{a}-1}
\end{gathered}
$$

Based on data extracted from the statistical community: $\chi=4, \mu 1=2, \mu 2=2, \mu 3=11, \mu 4=3, \mu 5=3.5, \mu 6=4, \mathrm{~m} 1=3$, $\mathrm{m} 2=1, \mathrm{~m} 3=\infty, \mathrm{m} 4=2, \mathrm{~m} 5=6, \mathrm{~m} 6=3, \mathrm{~B}=1.1, \mathrm{~K} 1=11, \mathrm{~K} 2=33, \mathrm{~K} 3=10$.

Therefore, 
Table 4. Q(u) Matrix

\begin{tabular}{|c|c|c|c|c|c|c|c|c|c|c|c|c|c|c|c|c|c|c|c|c|}
\hline ate & 1 & 2 & 3 & 4 & 5 & 6 & 7 & 8 & 9 & 10 & 11 & 12 & 13 & 14 & 15 & 16 & 17 & 18 & 19 & 20 \\
\hline 1 & & . & & 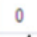 & 0 & 0 & 0 & 0 & 0 & 0 & 0 & 0 & 0 & 0 & 0 & 0 & 0 & 0 & 0 & 0 \\
\hline 2 & 0 & & 0 & $\gamma_{1}^{\prime}$ & $\gamma_{2}$ & 0 & 0 & 0 & 0 & 0 & 0 & 0 & 0 & 0 & 0 & 0 & 0 & 0 & 0 & 0 \\
\hline 3 & 0 & 0 & & 0 & $\gamma_{1}$ & $\gamma_{4}$ & 0 & 0 & 0 & 0 & 0 & 0 & 0 & 0 & 0 & 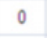 & 0 & 0 & 0 & 0 \\
\hline 4 & 0 & 0 & 0 & & 0 & 0 & $\gamma_{3}$ & $\gamma$ & 0 & 0 & 0 & 0 & 0 & 0 & 0 & 0 & 0 & 0 & 0 & 0 \\
\hline 5 & 0 & 0 & 0 & 0 & & 0 & 0 & $\gamma_{1}^{\prime}$ & $\gamma_{4}$ & 0 & 0 & 0 & 0 & 0 & 0 & 0 & 0 & 0 & 0 & 0 \\
\hline 6 & 0 & 0 & 0 & 0 & 0 & & 0 & 0 & $\gamma_{1}$ & $\gamma_{4}^{\prime}$ & 0 & 0 & 0 & 0 & 0 & 0 & 0 & 0 & 0 & 0 \\
\hline 7 & 0 & 0 & 0 & 0 & 0 & 0 & & 0 & 0 & 0 & $\gamma_{2}$ & 0 & 0 & 0 & 0 & 0 & 0 & 0 & 0 & 0 \\
\hline 8 & 0 & 0 & 0 & 0 & 0 & 0 & 0 & & 0 & 0 & $\gamma_{3}$ & $\gamma$ & 0 & 0 & 0 & 0 & 0 & 0 & 0 & 0 \\
\hline 9 & 0 & 0 & 0 & 0 & 0 & 0 & 0 & 0 & & 0 & 0 & $\gamma_{1}^{\prime}$ & $?$ & 0 & 0 & 0 & 0 & 0 & 0 & 0 \\
\hline 10 & 0 & 0 & 0 & 0 & 0 & 0 & 0 & 0 & 0 & & 0 & . & $\gamma_{1}$ & 0 & 0 & 0 & 0 & 0 & 0 & 0 \\
\hline 11 & 0 & 0 & 0 & 0 & 0 & 0 & 0 & 0 & 0 & 0 & & 0 & 0 & $\gamma_{4}$ & 0 & 0 & 0 & 0 & 0 & 0 \\
\hline 12 & 0 & 0 & 0 & 0 & 0 & 0 & 0 & 0 & 0 & 0 & 0 & & 0 & $\gamma_{3}$ & $\gamma_{4}^{\prime}$ & 0 & 0 & 0 & 0 & 0 \\
\hline 13 & 0 & 0 & 0 & 0 & 0 & 0 & 0 & 0 & 0 & 0 & 0 & 0 & & 0 & $\gamma_{1}^{\prime}$ & 0 & 0 & 0 & 0 & 0 \\
\hline 14 & 0 & 0 & 0 & 0 & 0 & 0 & 0 & 0 & 0 & 0 & 0 & 0 & 0 & & 0 & $\gamma_{4}^{\prime}$ & 0 & 0 & 0 & 0 \\
\hline 15 & 0 & 0 & 0 & 0 & 0 & 0 & 0 & 0 & 0 & 0 & 0 & 0 & 0 & 0 & & $\gamma_{3}$ & 0 & 0 & 0 & 0 \\
\hline 16 & 0 & 0 & 0 & 0 & 0 & 0 & 0 & 0 & 0 & 0 & 0 & 0 & 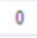 & 0 & 0 & & $\gamma_{s}$ & 0 & 0 & 0 \\
\hline 17 & 0 & 0 & 0 & 0 & 0 & 0 & 0 & 0 & 0 & 0 & 0 & 0 & 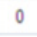 & 0 & 0 & 0 & & $\gamma_{s}^{\prime}$ & 0 & 0 \\
\hline 18 & 0 & 0 & 0 & 0 & 0 & 0 & 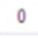 & 0 & 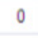 & 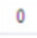 & 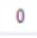 & 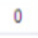 & 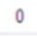 & 0 & 0 & 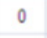 & 0 & & $y_{6}$ & 0 \\
\hline 19 & 0 & 0 & 0 & 0 & 0 & 0 & 0 & 0 & 0 & 0 & 0 & 0 & 0 & 0 & 0 & 0 & 0 & 0 & & $\gamma_{6}^{\prime}$ \\
\hline 20 & 0 & 0 & 0 & 0 & 0 & 0 & 0 & 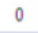 & 0 & 0 & . & 0 & 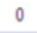 & 0 & 0 & 0 & 0 & 0 & 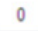 & \\
\hline
\end{tabular}

After solving the differential equation with use of Eq.5, Cumulative function of project completion time is follow:

$\mathrm{F}(\mathrm{t})=5.321 e^{-27 t}-17.136 e^{-6 t}-0.92 e^{-44 t}-3.1 e^{-9 t}+0.829 e^{-24 t}+1.9 e^{-33 t}-2.714 e^{-t}-$ $2.198 e^{-27 t}+2.323 e^{-10 t}-0.004 e^{-19 t}+0.34 e^{-12 t}+48.617 e^{-5 t}+1.1 e^{-5 t}+2.865 e^{-8 t}-0.002$ $e^{-33 t}-16.987 e^{-29 t}+1$

Based on algorithm:

$\mathrm{F}(\mathrm{B})=0.311<((\mathrm{K} 2-\mathrm{K} 1) /(\mathrm{K} 2+\mathrm{K} 3))=0.517$ then $\mathrm{t}^{*}=1.8(\mathrm{~F}(1.8)=0.517)$

That Due Date Assignment for Delivery Time for each project is Lag Project plus 1.8 year.

\section{Conclusion}

This study argued about analytical approximation of distribution function of project and mean project completion time. A chance-constrained programming developed by Charnes et al.[4]. Based on results, they have assumed exponential activity durations. Analytical procedure for PERT networks using continuous-time Markov chains with independent and exponentially distributed activity durations developed by Kulkarni and Adlakha[11] Hardie [10] has used Markov chain to activity durations that were not independent.

Based on results of estimate the project completed time, Fatemi et al [8] presented a structural mechanism with combined Seri and parallel mechanism. Schmit and Grossmann [18] developed a new technique for computing the project exact time project and defined over a finite interval while action time duration applied a term density function which combined Dirac delta functions with piecewise polynomial segments. Evaluating time of project putting into to relate project time and every possible PERT-path were introduced by Pontrandolfo[17].

In this paper, to estimate a dynamic method to procedure length maximum to accomplished the process of the dynamic Markov PERT network node, we have designed a procedure according to combined semi-Markovian processes and graph theory. Based on required time for finishing project, a lower bound is specified according its length.

In order to obtain a fixed time project delivery deadlines in dynamic PERT networks with multiple servers, the network may become a network queue, creating a suitable Markov models with finite state and continuous time then, differential equation system could be established and finally support the completed time project of distribution function. With appraising the completed time project of distribution function of dynamic PERT networks, or a start to develop some analysis it can be estimate less limitations as for the project scheduling.

\section{References}

Abdelkader, Y. (2004). Evaluating project completion times when activity times are Weibull distributed. European Journal of Operational Research, 157, 704-715. 
Akbari, R., Zeigham, V., \& Ziarati, K. (2011). Artificial Bee Colony for Resource Constrained Project Scheduling Problem. International Journal of Industrial Engineering computations, 2, 45-60.

Azaron, A., \& Ghomi, F. (2008). Lower bound for the mean project completion time in dynamic PERT networks. European Journal of Operational Research, 186, 120-127.

Charnes, W., Cooper, G., \& Thompson (1964). Critical path analysis via chance constrained and stochastic programming. Operations Research, 12, 460-470.

Chen, P. H., \& Shahandashti, S. M. (2009). Hybrid of Genetic Algorithm and Simulated Annealing for Multiple Project Scheduling with Multiple Resource Constraints. Automation in Construction, 18, 434-443.

Cohen, I., Golany, B., \& Shtub, A. (2007). Resource Allocation in Stochastic, Finite-Capacity, Multi-Project Systems through the Cross Entropy Methodology. Journal of Scheduling, 10, 181-193.

Elmaghraby, S. (2005). On the fallacy of averages in project risk management. European Journal of Operational Research, 165, 307-313.

Fatemi, G. S., \& Rabbani, M. (2003). A new structural mechanism for reducibility of stochastic PERT networks. European Journal of Operational Research, 145, 394-402.

Goncalves, J. F., Mendes, J. J. M., \& Resende, M. G. C. (2008). A Genetic Algorithm for the Resource Constrained Multi-Project Scheduling Problem. European Journal of Operational Research, 189, 11711190.

Hardie, N. (2001). The prediction and control of project duration: A recursive model. International Journal of Project Management, 19, 401-409.

Kulkarni, V., \& Adlakha, V. (1986). Markov and Markov-regenerative PERT networks. Operations Research, 34, 769-781.

Kumanan, S., Jegan, J. G., \& Raja, K. (2006). Multi-Project Scheduling using an Heuristic and a Genetic Algorithm. The International Journal of Advanced Manufacturing Technology, 31, 360- 366.

Lachmayer, R., \& Afsari, M. (2012). Algorithm of Converting Fuzzy GERT into CPM for Research Project Scheduling With Case Study, 8th International Conference of Project Management, Tehran, Iran.

Lachmayer, R., \& Afsari, M. (2013). Fuzzy GERT method for Scheduling Research Projects, 9th International Conference of Industrial Engineering, Tehran, Iran.

Lova, A., Maroto, C., \& Tormos, P. (2000). A Multicriteria Heuristic Method to Improve Resource Allocation in Multiproject Scheduling. European Journal of Operational Research, 127, 408-424.

Ludwig, A., Mohring, R. H., \& Frederik, S. (2001). A Computational Study on Bounding the Makespan Distribution in Stochastic Project Networks. Annals of Operations Research, 102, 49-64.

Pontrandolfo, P., (2000). Project duration in stochastic networks by the PERT-path technique. International Journal of Project Management, , 215-222.

Schmit, I., \& Grossmann (2000). The exact overall time distribution of a project with uncertain task durations. European Journal of Operational Research, 126, 614-636.

Yaghoubi, S., Noori, S., Azaron A., \& Tavakkoli-Moghaddam, R. (2011). Resource Allocation in Dynamic PERT Networks with Finite Capacity. European Journal of Operational Research, 215, 670-678.

Ying, Y., Shou, Y., \& Li, M. (2009). Hybrid Genetic Algorithm for Resource Constrained Multi-Project Scheduling Problem, Journal of Zhejiang University (Engineering Science), 43, 23-27.

\section{Copyrights}

Copyright for this article is retained by the author(s), with first publication rights granted to the journal.

This is an open-access article distributed under the terms and conditions of the Creative Commons Attribution license (http://creativecommons.org/licenses/by/3.0/). 